\begin{tabular}{c|c|c|c|} 
SELECCIONES MATEMÁTICAS \\
Universidad Nacional de Trujillo \\
ISSN: $2411-1783$ (Online) \\
2021; Vol. 8(1): 37-51.
\end{tabular}

\title{
Existence and Regularity of the Solution of Non homogeneous Schrödinger Equation in Periodic Sobolev Spaces
}

\section{Existencia y Regularidad de Solución de la Ecuación de Schrödinger no Homogénea en Espacios de Sobolev Periódico}

\author{
Yolanda Santiago Ayala ${ }^{\circledR}$ and Santiago Rojas Romero
}

Received, Nov. 04, 2020

Accepted, May. 12, 2021

How to cite this article:

Santiago Y, Rojas S. Existence and Regularity of the Solution of Non homogeneous Schrödinger Equation in Periodic Sobolev Spaces. Selecciones Matemáticas. 2021;8(1):37-51. http://dx.doi.org/10.17268/sel .mat. 2021.01 .04

\begin{abstract}
In this article we prove that the Cauchy problem associated to the Schrödinger equation in periodic Sobolev spaces is well posed. We do this in an intuitive way using Fourier theory and in a fine version using Groups theory, inspired by works Iorio [3], Santiago and Rojas [12] and [13]. Also, we study the relationship between initial data and differentiability of the solution.

Finally, we study the corresponding non-homogeneous problem and prove that it is locally well posed, and that the solution has continuous dependence with respect to the initial data and the non-homogeneity in compact intervals.

Keywords. Groups theory, Schrödinger equation, non homogeneous equation, periodic Sobolev spaces, Fourier theory.
\end{abstract}

\section{Resumen}

En este artículo probamos que el problema de Cauchy asociado a la ecuación de Schrödinger en espacios de Sobolev periódico está bien colocado. Hacemos esto en un modo intuitivo usando la teoría de Fourier y en una versión elegante usando la teoría de grupos, inspirados en los trabajos de Iorio [3], Santiago and Rojas [12] y [13]. También, estudiamos la relación entre el dato inicial y la diferenciabilidad de la solución.

Finalmente, estudiamos el correspondiente problema no homogéneo y probamos que está localmente bien colocado, y que la solución posee dependencia continua respecto al dato inicial y a la no homogeneidad en intervalos compactos.

Palabras clave. Teoría de grupos, ecuación de Schrödinger, ecuación no homogénea, espacios de Sobolev periódico, teoría de Fourier.

1. Introducción. Sea la ecuación del Schrödinger propuesto por el físico austriaco Erwin Schrödinger (1925)

$$
u_{t}-i \alpha^{2} u_{x x}=0
$$

con dato inicial $u(0)=\phi \in H_{\text {per }}^{s}$, donde $s$ es un número real y denotamos por $H_{\text {per }}^{s}$ al espacio de Sobolev periódico de orden $s$. Dicha ecuación describe la evolución temporal de una partícula no relativista.

\footnotetext{
*Facultad de Ciencias Matemáticas, Universidad Nacional Mayor de San Marcos, Av. Venezuela S/N Lima 01, Lima-Perú. (ysantiagoa@unmsm. edu.pe, rsernad@unmsm. edu.pe).

${ }^{\dagger}$ Facultad de Ciencias Matemáticas, Universidad Nacional Mayor de San Marcos, Av. Venezuela S/N Lima 01, Lima-Perú. (srojasreunmsm. edu.pe).
} 
Esta ecuación es importante en la teoría de la mecánica cuántica. Schrödinger discute en detalle las relaciones entre la mecánica hamiltoniana y óptica en 1926, ver [6] y [14]. Para la justificación física del modelo podemos citar [2] y [15].

De [2], la ecuación de Schrödinger homogénea está bien colocada para todo $s$ real. Este resultado lo demostraremos adaptando y siguiendo las ideas de [3], [12] y [13].

Citamos algunos trabajos de existencia via semigrupos [5], [7], [8], [9], [10] y nos apoyamos de algunos resultados de [11]. También, no podemos dejar de mencionar la riqueza de información de [4] y [1].

Probaremos la existencia y unicidad de solución de (1.1), así como la dependencia continua de la solución respecto al dato inicial. Luego, introduciremos una familia de operadores para reescribir nuestro resultado en una versión más elegante. Haremos el análisis de diferenciabilidad versus dato inicial del problema.

Luego, surge naturalmente preguntarse ¿Qué sucede para el caso no homogéneo? ¿Será bien colocado?

Bueno, motivados por [3] y [13] podremos responder las interrogantes planteadas. Esto es, probaremos que el modelo no homogéneo de (1.1) está localmente bien colocado.

Nuestro artículo está organizado del siguiente modo. En la sección 2, indicamos la metodología usada y citamos la referencia usada para los resultados preliminares que se puedan necesitar. En la sección 3, probamos que el problema de Cauchy asociado a la ecuación de Schrödinger homogéneo está bien colocado. En la sección 4, hacemos el análisis de la diferenciabilidad de la solución versus dato inicial. En la sección 5, probamos que el problema de Cauchy asociado a la ecuación de Schrödinger no homogénea está localmente bien colocada y además obtenemos que la solución depende continuamente respecto al dato inicial y a la parte no homogénea del problema.

Finalmente, en la sección 6, damos las conclusiones de nuestro estudio.

2. Metodología. Como marco teórico, en este trabajo usamos fuertemente los siguientes tópicos: Teoría de Fourier en espacios de Sobolev periódico, análisis armónico, teoría de grupos, semigrupos de clase $C_{o}$ y familias fuertemente continuas. Como referencia en la revisión de algunos resultados previos que usaremos, citamos a Iorio [3], Santiago and Rojas [12, 13].

Toda esta teoría la usamos en el análisis de existencia y buena colocación del problema de Cauchy para la ecuación de Schrödinger, realizando una serie de cálculos y aproximaciones en el desarrollo del trabajo.

3. Existencia de solución de la ecuación de Schrödinger homogénea . En esta sección, empezamos probando que existe solución de la ecuación de Schrödinger homogénea en espacios de Sobolev Periódico, usando la teoría de Fourier.

Teorema 3.1. Sea s un número real fijo y el problema homogéneo

$$
\left(P_{3}\right) \mid \begin{aligned}
& u \in C\left(\mathbb{R}, H_{p e r}^{s}\right) \cap C^{1}\left(\mathbb{R}, H_{p e r}^{s-2}\right), \\
& \partial_{t} u-i \partial_{x}^{2} u=0 \in H_{p e r}^{s-2} \\
& u(0)=\phi \in H_{p e r}^{s},
\end{aligned}
$$

entonces $\left(P_{3}\right)$ está globalmente bien colocado i.e. $\exists ! u \in C\left(\mathbb{R}, H_{\text {per }}^{s}\right) \cap C^{1}\left(\mathbb{R}, H_{\text {per }}^{s-2}\right)$ satisfaciendo la ecuación $\left(P_{3}\right)$, de modo que la aplicación $: \phi \rightarrow u$, que asigna a cada dato inicial $\phi$ la solución u del PVI $\left(P_{3}\right)$, es continua.

Además, la solución u satisface la regularidad: $u(t) \in H_{p e r}^{r}, \forall t \in \mathbb{R}, \forall r \leq s$ con $\|u(t)\|_{H_{p e r}^{r}} \leq\|\phi\|_{H_{p e r}^{s}}$, $\forall t \in \mathbb{R}, \forall r<s$ y $\|u(t)\|_{H_{\text {per }}^{s}}=\|\phi\|_{H_{\text {per }}^{s}}, \forall t \in \mathbb{R}$.

También se obtiene que la aplicación: $\phi \rightarrow \partial_{t}$ u que asigna a cada dato inicial $\phi$ la derivada de la solución $u$ del PVI $\left(P_{3}\right): \partial_{t} u$, es continua y satisface:

$$
\begin{aligned}
\left\|\partial_{t} u(t)-\partial_{t} \tilde{u}(t)\right\|_{s-2} & \leq\|\phi-\tilde{\phi}\|_{s}, \forall t \in \mathbb{R}, \\
\sup _{t \in \mathbb{R}}\left\|\partial_{t} u(t)-\partial_{t} \tilde{u}(t)\right\|_{s-2} & \leq\|\phi-\tilde{\phi}\|_{s} .
\end{aligned}
$$

Además, $\partial_{t} u(t) \in H_{\text {per }}^{\theta}, \forall t \in \mathbb{R}, \forall \theta \leq s-2$ y satisface:

$$
\begin{aligned}
\left\|\partial_{t} u(t)\right\|_{\theta} & \leq\|\phi\|_{s}, \forall t \in \mathbb{R}, \forall \theta \leq s-2, \\
\sup _{t \in \mathbb{R}}\left\|\partial_{t} u(t)\right\|_{\theta} & \leq\|\phi\|_{s}, \forall \theta \leq s-2 .
\end{aligned}
$$

Demostración: La prueba lo hacemos de la siguiente forma: 
1. Primero obtendremos el candidato a solución. Para conseguir ese candidato tomamos la transformada de Fourier a la ecuación

$$
\partial_{t} u-i \partial_{x}^{2} u=0
$$

obteniendo

$$
0=\partial_{t} \hat{u}-i(i k)^{2} \hat{u}=\partial_{t} \hat{u}+i k^{2} \hat{u},
$$

que para cada $k \in \mathbb{Z}$ es una EDO con dato inicial $\hat{u}(k, 0)=\hat{\phi}(k)$.

Así, planteamos un sistema no acoplado de ecuaciones diferenciales ordinarias de primer orden homogéneo

$$
\left(\Omega_{k}\right) \mid \begin{aligned}
& \hat{u} \in C\left(\mathbb{R}, l_{s}^{2}(\mathbb{Z})\right), \\
& \partial_{t} \hat{u}(k, t)+i k^{2} \hat{u}(k, t)=0, \\
& \hat{u}(k, 0)=\hat{\phi}(k) \operatorname{con} \widehat{\phi} \in l_{s}^{2}(\mathbb{Z}),
\end{aligned}
$$

$\forall k \in \mathbb{Z}$ y conseguimos

$$
\hat{u}(k, t)=e^{-i k^{2} t} \hat{\phi}(k),
$$

de donde obtenemos nuestro candidato a solución:

$$
u(t)=\sum_{k=-\infty}^{\infty} \hat{u}(k, t) \phi_{k}=\sum_{k=-\infty}^{\infty} \underbrace{e^{-i k^{2} t}}_{|\cdot|=1} \hat{\phi}(k) \phi_{k} .
$$

2. En segundo lugar, probaremos que

$$
u(t) \in H_{\text {per }}^{s} \mathrm{y}\|u(t)\|_{s}=\|\phi\|_{s}, \forall t \in \mathbb{R} .
$$

En efecto, sea $t \in \mathbb{R}$ y $\phi \in H_{\text {per }}^{s}$, entonces

$$
\begin{aligned}
\|u(t)\|_{H_{p e r}^{s}}^{2} & =2 \pi \sum_{k=-\infty}^{+\infty}\left(1+k^{2}\right)^{s}\left|e^{-i k^{2} t} \hat{\phi}(k)\right|^{2} \\
& =2 \pi \sum_{k=-\infty}^{+\infty}\left(1+k^{2}\right)^{s}|\hat{\phi}(k)|^{2}, \\
& =\|\phi\|_{H_{p e r}^{s}}^{2} .
\end{aligned}
$$

3. Ahora, probaremos que $u(\cdot)$ es continua en $\mathbb{R}$.

Sea $t^{\prime} \in \mathbb{R}$, obtenemos

$$
\begin{aligned}
\left\|u(t)-u\left(t^{\prime}\right)\right\|_{H_{p e r}^{s}}^{2} & =2 \pi \sum_{k=-\infty}^{+\infty}\left(1+k^{2}\right)^{s}\left|\left(e^{-i k^{2} t}-e^{-i k^{2} t^{\prime}}\right) \hat{\phi}(k)\right|^{2} \\
& =2 \pi \sum_{k=-\infty}^{+\infty}\left(1+k^{2}\right)^{s}|\hat{\phi}(k)|^{2}|\underbrace{e^{-i k^{2} t}-e^{-i k^{2} t^{\prime}}}_{H(t):=}|^{2} .
\end{aligned}
$$

Se observa que $\lim _{t \rightarrow t^{\prime}} H(t)=0$. Ahora, necesitamos de la convergencia uniforme de la serie para el intercambio de límites. Para esto, tomamos el k-ésimo término de la serie y lo mayoramos por una serie convergente. Esto es,

$$
I_{k, t}:=2 \pi\left(1+k^{2}\right)^{s}|\hat{\phi}(k)|^{2} \underbrace{\left|e^{-i k^{2} t}-e^{-i k^{2} t^{\prime}}\right|^{2}}_{\leq 2} \leq 8 \pi\left(1+k^{2}\right)^{s}|\hat{\phi}(k)|^{2},
$$

donde hemos usado la desigualdad triangular (propiedad de la norma) y la igualdad $\left|e^{i \theta}\right|=1$, $\forall \theta \in \mathbb{R}$.

Así,

$$
\sum_{k=-\infty}^{+\infty} I_{k, t} \leq 4\|\phi\|_{H_{p e r}^{s}}^{2}<\infty
$$


y usando el Teorema del M-Test de Weierstrass tenemos que la serie converge uniformemente. Luego, está permitido el intercambio de límite, esto es,

$$
0 \leq \lim _{t \rightarrow t^{\prime}}\left\|u(t)-u\left(t^{\prime}\right)\right\|_{H_{p e r}^{s}}^{2}=\sum_{k=-\infty}^{+\infty} \underbrace{\lim _{t \rightarrow t^{\prime}} I_{k, t}}_{=0}=0
$$

y de ahí concluimos $\lim _{t \rightarrow t^{\prime}}\left\|u(t)-u\left(t^{\prime}\right)\right\|_{H_{p e r}^{s}}=0$.

4. Probaremos que

$$
\left\|\frac{u(t+h)-u(t)}{h}-i \partial_{x}^{2} u\right\|_{H_{p e r}^{s-2}} \longrightarrow 0 \quad \text { cuando } h \rightarrow 0 .
$$

En efecto,

$$
\left\|\frac{u(t+h)-u(t)}{h}-i \partial_{x}^{2} u\right\|_{H_{\text {per }}^{s-2}}^{2}
$$

$$
\begin{aligned}
& =2 \pi \sum_{k=-\infty}^{+\infty}\left(1+k^{2}\right)^{s-2}|\hat{\phi}(k)|^{2}\left|\frac{e^{-i k^{2}(t+h)}-e^{-i k^{2} t}}{h}-i(i k)^{2} e^{-i k^{2} t}\right|^{2} \\
& =2 \pi \sum_{k=-\infty}^{+\infty}\left(1+k^{2}\right)^{s-2}|\hat{\phi}(k)|^{2}|e^{-i k^{2} t} \cdot \underbrace{\left\{\frac{e^{-i k^{2} h}-1}{h}+i k^{2}\right\}}_{M(h):=}|^{2} .
\end{aligned}
$$

Usando L'Hospital tenemos que $M(h) \longrightarrow 0$ cuando $h \rightarrow 0$.

Ahora, necesitamos la convergencia uniforme de la serie para habilitar el intercambio de límites. Para ello procedemos mayorando el k-ésimo término de la serie. Previamente, para $h>0$ observamos lo siguiente:

$$
\frac{e^{-i k^{2} h}-1}{h}=\int_{0}^{h} \frac{1}{h} \frac{\partial}{\partial s}\left\{e^{-i k^{2} s}\right\} d s=\int_{0}^{h} \frac{1}{h}\left[-i k^{2}\right] e^{-i k^{2} s} d s
$$

y tomando norma, tenemos

$$
\left|\frac{e^{-i k^{2} h}-1}{h}\right| \leq \frac{1}{h}\left|-i k^{2}\right| \int_{0}^{h}\left|e^{-i k^{2} s}\right| d s=\frac{1}{h}\left\{|k|^{2}\right\} \cdot h=|k|^{2} .
$$

Considerando $h<0$ para el caso $t \neq 0$, obtenemos

$$
\frac{e^{-i k^{2} h}-1}{h}=-\int_{h}^{0} \frac{1}{h} \frac{\partial}{\partial s}\left\{e^{-i k^{2} s}\right\} d s=-\int_{h}^{0} \frac{1}{h}\left[-i k^{2}\right] e^{-i k^{2} s} d s,
$$

tomando norma y usando que $\left|e^{i \vartheta}\right|=1, \forall \vartheta \in \mathbb{R}$, tenemos

$$
\left|\frac{e^{-i k^{2} h}-1}{h}\right| \leq \frac{1}{|h|}\left|i k^{2}\right| \int_{h}^{0} d s=\frac{1}{|h|}\left\{|k|^{2}\right\} \cdot|h|=|k|^{2} .
$$

Usando las desigualdades (3.6) y (3.7) procedemos a mayorar $|M(h)|^{2}$ como sigue

$$
|M(h)|^{2} \leq\left\{2|k|^{2}\right\}^{2} \leq C_{5}\left[1+|k|^{2}\right]^{2} .
$$

Esto también es válido para el caso $t=0$, donde hemos usado (3.6) para el caso $h>0$ y (3.7) para el caso $h<0$.

Ahora, pasamos a mayorar el k-ésimo término de la serie, donde usamos la estimativa (3.8), obteniendo

$$
\begin{aligned}
\left(1+k^{2}\right)^{s-2}|\hat{\phi}(k)|^{2}|M(h)|^{2} & \leq\left(1+k^{2}\right)^{s-2}|\hat{\phi}(k)|^{2} C_{5}\left(1+|k|^{2}\right)^{2} \\
& =C_{5}\left(1+k^{2}\right)^{s}|\hat{\phi}(k)|^{2}
\end{aligned}
$$


y como la serie $2 \pi \sum_{k=-\infty}^{+\infty}\left(1+k^{2}\right)^{s}|\hat{\phi}(k)|^{2}=\|\phi\|_{H_{p e r}^{s}}^{2}<\infty$ desde que $\phi \in H_{p e r}^{s}$, entonces usando el Teorema M-Test de Weierstrass tenemos que la serie (3.5) converge uniformemente y por lo tanto es posible intercambiar límites y obtener

$$
\left\|\frac{u(t+h)-u(t)}{h}-i \partial_{x}^{2} u\right\|_{H_{\text {per }}^{s-2}}^{2} \longrightarrow 0 \quad \text { cuando } h \rightarrow 0 .
$$

5. Probaremos la dependencia continua de la solución respecto a los datos iniciales, i.e. sean $\phi \mathrm{y}$ $\widetilde{\phi}$ datos próximos en $H_{\text {per }}^{s}$, entonces sus correspondientes soluciones $u$ y $\widetilde{u}$, respectivamente, también están próximos en el espacio solución. Sea $t \in \mathbb{R}$,

$$
\begin{aligned}
\|u(t)-\widetilde{u}(t)\|_{H_{p e r}^{s}}^{2} & =2 \pi \sum_{k=-\infty}^{+\infty}\left|e^{-i k^{2} t}(\widehat{\phi}(k)-\widehat{\widetilde{\phi}}(k))\right|^{2}\left(1+k^{2}\right)^{s} \\
& =2 \pi \sum_{k=-\infty}^{+\infty}\left(1+k^{2}\right)^{s}|\widehat{\phi}(k)-\widehat{\widetilde{\phi}}(k)|^{2} \\
& =\|\phi-\widetilde{\phi}\|_{H_{p e r}^{s}}^{2} .
\end{aligned}
$$

Tomando supremo sobre $\mathbb{R}$, tenemos

$$
\sup _{t \in \mathbb{R}}\|u(t)-\widetilde{u}(t)\|_{H_{p e r}^{s}}=\|\phi-\widetilde{\phi}\|_{H_{p e r}^{s}} .
$$

De aquí tenemos que si $\phi \rightarrow \widetilde{\phi}$ entonces $u \rightarrow \widetilde{u}$.

6. Unicidad de Solución.- La igualdad (3.9) nos permitirá mostrar que la solución es única. En efecto, sea $\phi \in H_{\text {per }}^{s}$ y supongamos que existan $u$ y $\widetilde{u}$ dos soluciones, usando (3.9) obtenemos,

$$
\|u(\tau)-\widetilde{u}(\tau)\|_{H_{p e r}^{s}} \leq \sup _{t \in \mathbb{R}}\|u(t)-\widetilde{u}(t)\|_{H_{p e r}^{s}}=\|\phi-\widetilde{\phi}\|_{H_{p e r}^{s}}=0, \forall \tau \in \mathbb{R},
$$

de donde concluimos que $u=\tilde{u}$.

Así, el problema $\left(P_{3}\right)$ está bien colocado y su única solución que depende continuamente del dato inicial es

$$
u(x, t)=\sum_{k=-\infty}^{+\infty} e^{-i k^{2} t} \hat{\phi}(k) e^{i k x} .
$$

7. Sea $r<s$ entonces $H_{p e r}^{s} \subset H_{p e r}^{r}$ y desde que el dato inicial $\phi \in H_{p e r}^{s}$, entonces $\phi \in H_{p e r}^{r}$ y satisface

$$
\|\phi\|_{r} \leq\|\phi\|_{s} .
$$

De (3.3) y usando (3.10) obtenemos

$$
\|u(t)\|_{r}^{2}=\|\phi\|_{r}^{2} \leq\|\phi\|_{s}^{2}<\infty .
$$

Es decir,

$$
u(t) \in H_{p e r}^{r}, \forall r \in(-\infty, s) .
$$

8. Así, de (3.2) y (3.11) concluimos que para $t \in \mathbb{R}$ se tiene

$$
u(t) \in H_{p e r}^{r}, \forall r \in(-\infty, s] .
$$

9. Probaremos que $\partial_{t} u(\cdot)$ es continua en $\mathbb{R}$. En efecto, sea $t \in \mathbb{R}$, conseguimos:

$$
\begin{aligned}
& \left\|\partial_{t} u(t)-\partial_{t} u\left(t^{\prime}\right)\right\|_{s-2}^{2} \\
& =2 \pi \sum_{k=-\infty}^{+\infty}\left(1+k^{2}\right)^{s-2} \widehat{\mid \partial_{t} u(t)}(k)-\left.\widehat{\partial_{t} u\left(t^{\prime}\right)}(k)\right|^{2} \\
& =2 \pi \sum_{k=-\infty}^{+\infty}\left(1+k^{2}\right)^{s-2}\left|\left(i k^{2}\right)\left\{e^{-i k^{2} t}-e^{-i k^{2} t^{\prime}}\right\} \widehat{\phi}(k)\right|^{2} \\
& =2 \pi \sum_{k=-\infty}^{+\infty}\left(1+k^{2}\right)^{s-2}\left(k^{2}\right)^{2} \underbrace{\left|e^{-i k^{2} t}-e^{-i k^{2} t^{\prime}}\right|^{2}}_{H(t):=}|\widehat{\phi}(k)|^{2} \\
& \leq \sum_{k=-\infty}^{+\infty} \underbrace{2 \pi\left(1+k^{2}\right)^{s} H(t)|\widehat{\phi}(k)|^{2}}_{I_{k, t}:=} .
\end{aligned}
$$


Se observa que $\lim _{t \rightarrow t^{\prime}} H(t)=0$.

Ahora, necesitamos de la convergencia uniforme de la serie para el intercambio de límites. Para esto, tomamos el $k$ - ésimo término de la serie y lo mayoramos por una serie convergente, i.e.

$$
I_{k, t} \leq 2 \pi\left(1+k^{2}\right)^{s} 4|\widehat{\phi}(k)|^{2}=8 \pi\left(1+k^{2}\right)^{s}|\widehat{\phi}(k)|^{2},
$$

donde hemos usado la desigualdad triangular (propiedad de la norma) y la igualdad $\left|e^{i \theta}\right|=1$, $\forall \theta \in \mathbb{R}$.

Así, la serie

$$
\sum_{k=-\infty}^{+\infty} I_{k, t} \leq 4\|\phi\|_{s}^{2}<\infty
$$

es convergente, y usando el Teorema del M-Test de Weierstras tenemos que la serie converge uniformemente. Luego está permitido el intercambio de límite, esto es

$$
0 \leq \lim _{t \rightarrow t^{\prime}}\left\|\partial_{t} u(t)-\partial_{t} u\left(t^{\prime}\right)\right\|_{s-2}^{2}=\sum_{k=-\infty}^{+\infty} \underbrace{\lim _{t \rightarrow t^{\prime}} I_{k, t}}_{=0}=0
$$

y de ahí concluimos

$$
\lim _{t \rightarrow t^{\prime}}\left\|\partial_{t} u(t)-\partial_{t} u\left(t^{\prime}\right)\right\|_{s-2}^{2}=0
$$

Esto es, $\lim _{t \rightarrow t^{\prime}}\left\|\partial_{t} u(t)-\partial_{t} u\left(t^{\prime}\right)\right\|_{s-2}=0$.

10. Probaremos la dependencia continua de $\partial_{t} u$ respecto a los datos iniciales, i.e sean $\phi$ y $\widetilde{\phi}$ próximos en $H_{p e r}^{s}$, entonces sus correspondientes derivadas de las soluciones $u \mathrm{y} \widetilde{u}$, esto es $\partial_{t} u \mathrm{y}$ $\partial_{t} \widetilde{u}$, respectivamente, también están próximos. Sea $t \in \mathbb{R}$,

$$
\begin{aligned}
\left\|\partial_{t} u(t)-\partial_{t} \widetilde{u}(t)\right\|_{s-2}^{2} & =2 \pi \sum_{k=-\infty}^{+\infty}\left(1+k^{2}\right)^{s-2}\left|\left(-i k^{2}\right) e^{-i k^{2} t}\{\widehat{\phi}(k)-\widehat{\widetilde{\phi}}(k)\}\right|^{2} \\
& =2 \pi \sum_{k=-\infty}^{+\infty}\left(1+k^{2}\right)^{s-2}\left(k^{4}\right)|\widehat{\phi}(k)-\widehat{\widetilde{\phi}}(k)|^{2} \\
& \leq 2 \pi \sum_{k=-\infty}^{+\infty}\left(1+k^{2}\right)^{s}|\widehat{\phi}(k)-\widehat{\widetilde{\phi}}(k)|^{2} \\
& =\|\phi-\widetilde{\phi}\|_{s}^{2}
\end{aligned}
$$

esto es,

$$
\left\|\partial_{t} u(t)-\partial_{t} \widetilde{u}(t)\right\|_{s-2}^{2} \leq\|\phi-\widetilde{\phi}\|_{s}^{2} .
$$

Así,

$$
\left\|\partial_{t} u(t)-\partial_{t} \widetilde{u}(t)\right\|_{s-2} \leq\|\phi-\widetilde{\phi}\|_{s} .
$$

Tomando supremo sobre $\mathbb{R}$, obtenemos

$$
\sup _{t \in \mathbb{R}}\left\|\partial_{t} u(t)-\partial_{t} \tilde{u}(t)\right\|_{s-2} \leq\|\phi-\widetilde{\phi}\|_{s} .
$$

11. Del item anterior tenemos que vale la desigualdad

$$
\left\|\partial_{t} u(t)\right\|_{s-2} \leq\|\phi\|_{s} .
$$

Además, usando inmersión continua de espacios de Sobolev periódico, tenemos que $\left\|\partial_{t} u(t)\right\|_{r-2} \leq$ $\|\phi\|_{r} \leq\|\phi\|_{s}, \forall r<s$. Así, $\partial_{t} u(t) \in H_{p e r}^{\theta}, \forall \theta \in(-\infty, s-2]$.

En consecuencia tenemos el siguiente resultado.

Corolario 3.1. La única solución de $\left(P_{3}\right)$ es

$$
u(x, t)=\sum_{k=-\infty}^{+\infty} e^{-i k^{2} t} \hat{\phi}(k) e^{i k x}
$$


Ahora, introduciremos una familia de operadores que verificarán las condiciones de ser un grupo de clase $C_{0}$.

Teorema 3.2. Sea $s \in \mathbb{R}$ y la aplicación

$$
\begin{aligned}
T: \mathbb{R} & \rightarrow L\left(H_{\text {per }}^{s}\right) \\
t & \rightarrow T(t)
\end{aligned}
$$

tal que $T(t)=e^{i \partial_{x}^{2} t}$, i.e. aplica $T(t) \phi=\left\{e^{-i k^{2} t} \hat{\phi}(k)\right\}^{\vee}, \forall \phi \in H_{\text {per }}^{s}$. Entonces $\{T(t)\}_{t \in \mathbb{R}}$ es un grupo unitario de clase $C_{0}$ en $H_{\text {per }}^{s}$.

Además, se verifican los siguientes enunciados:

1. $T(\cdot) \phi \in C\left(\mathbb{R}, H_{\text {per }}^{s}\right), \forall \phi \in H_{\text {per }}^{s}$,

2. La aplicación $\phi \rightarrow T(\cdot) \phi$ es continua $y \forall \varphi_{1}, \varphi_{2} \in H_{\text {per }}^{s}$ se satisface:

$$
\begin{aligned}
\left\|T(t) \varphi_{1}-T(t) \varphi_{2}\right\|_{H_{p e r}^{s}} & =\left\|\varphi_{1}-\varphi_{2}\right\|_{H_{p e r}^{s}}, \forall t \in \mathbb{R}, \\
\sup _{t \in \mathbb{R}}\left\|T(t) \varphi_{1}-T(t) \varphi_{2}\right\|_{H_{p e r}^{s}} & =\left\|\varphi_{1}-\varphi_{2}\right\|_{H_{p e r}^{s}} .
\end{aligned}
$$

Demostración: Primero observamos que $T(0) \phi=\phi, \forall \phi \in H_{p e r}^{s}$, así $T(0)=I$. De la linealidad de la transformada de Fourier y de su inversa tenemos que $T(t)$ es lineal.

Si $\phi \in H_{\text {per }}^{s}$ probaremos que $T(t) \phi \in H_{\text {per }}^{s} \mathrm{y}\|T(t) \phi\|_{s}=\|\phi\|_{s}$, i.e. $\|T(t)\|=1$. En efecto, procediendo análogamente a (3.3), obtenemos

$$
\begin{aligned}
\|T(t) \phi\|_{H_{p e r}^{s}}^{2} & =2 \pi \sum_{k=-\infty}^{+\infty}\left(1+k^{2}\right)^{s}\left|e^{-i k^{2} t} \hat{\phi}(k)\right|^{2} \\
& =2 \pi \sum_{k=-\infty}^{+\infty}\left(1+k^{2}\right)^{s}|\hat{\phi}(k)|^{2} \\
& =\|\phi\|_{H_{p e r}^{s}}^{2}<\infty .
\end{aligned}
$$

Luego, $T(t) \phi \in H_{\text {per }}^{s} \mathrm{y}\|T(t) \phi\|_{s}=\|\phi\|_{s}$, es decir $T(t) \in L\left(H_{\text {per }}^{s}\right)$ con $\|T(t)\|=1$.

Ahora probaremos que $T(t+r)=T(t) \circ T(r), \forall t, r \in \mathbb{R}$. En efecto,

$$
T(t+r) f(x)=\sum_{k=-\infty}^{\infty} e^{-i k^{2}(t+r)} \hat{f}(k) e^{i k x}=\sum_{k=-\infty}^{\infty} e^{-i k^{2} t} \underbrace{e^{-i k^{2} r} \hat{f}(k)}_{\hat{g}(k):=} e^{i k x}=T(t) g(x),
$$

donde $g$ es tal que $\hat{g}(k)=e^{-i k^{2} r} \hat{f}(k), \forall k \in Z$. Así,

$$
g(x)=\sum_{k=-\infty}^{\infty} e^{-i k^{2} r} \hat{f}(k) e^{i k x}=T(r) f(x)
$$

Por lo tanto, $T(t+r) f=T(t) \circ T(r) f, \forall t, r \in \mathbb{R}$.

Ahora probaremos la continuidad de $t \rightarrow T(t) \phi$, esto es

$$
\|T(t+h) \phi-T(t) \phi\|_{H_{\text {per }}^{s}} \rightarrow 0 \text { cuando } h \rightarrow 0 .
$$

En efecto, usando el item 3 de la prueba del teorema anterior, tenemos

$$
\begin{aligned}
\| T(t+ & h) \phi-T(t) \phi \|_{H_{p e r}^{s}}^{2} \\
& =2 \pi \sum_{k=-\infty}^{+\infty}\left(1+k^{2}\right)^{s}\left|\left(e^{-i k^{2}(t+h)}-e^{-i k^{2} t}\right) \hat{\phi}(k)\right|^{2} \\
& =2 \pi \sum_{k=-\infty}^{+\infty}\left(1+k^{2}\right)^{s}|\hat{\phi}(k)|^{2}|\underbrace{e^{-i k^{2}(t+h)}-e^{-i k^{2} t}}_{H(t+h)}|^{2} .
\end{aligned}
$$

Observamos que $\lim _{h \rightarrow 0} H(t+h)=0$.

Ahora, necesitamos de la convergencia uniforme de la serie para el intercambio de límites. Para eso, tomamos el k-ésimo término de la serie y lo mayoramos por una serie convergente, i.e.

$$
I_{k, t, h}:=2 \pi\left(1+k^{2}\right)^{s}|\hat{\phi}(k)|^{2}\left|e^{-i k^{2}(t+h)}-e^{-i k^{2} t}\right|^{2} \leq 8 \pi\left(1+k^{2}\right)^{s}|\hat{\phi}(k)|^{2},
$$

donde hemos usado la desigualdad triangular (propiedad de la norma) y la igualdad $\left|e^{i \theta}\right|=1, \forall \theta \in \mathbb{R}$. Así,

$$
\sum_{k=-\infty}^{+\infty} I_{k, t, h} \leq 4\|\phi\|_{H_{p e r}^{s}}^{2}<\infty
$$


y usando el Teorema del M-Test de Weierstrass tenemos que la serie en (3.16) converge uniformemente. Luego está permitido el intercambio de límite, esto es

$$
\lim _{h \rightarrow 0}\|T(t+h) \phi-T(t) \phi\|_{H_{p e r}^{s}}^{2}=\sum_{k=-\infty}^{+\infty} \underbrace{\lim _{h \rightarrow 0} I_{k, t, h}}_{=0}=0 .
$$

Observación 3.1. Se verifica rápidamente que

$$
\lim _{t \rightarrow 0}\|T(t) \phi-\phi\|_{H_{p e r}^{s}}=0, \forall \phi \in H_{p e r}^{s} .
$$

Se sabe, lo siguiente:

Observación 3.2. Si $\{T(t)\}_{t \in \mathbb{R}}$ es un grupo de clase $C_{0}$ entonces satisface

$$
\lim _{t \rightarrow r}\|T(t) \phi-T(r) \phi\|_{H_{p e r}^{s}}=0 \forall r \in \mathbb{R}, \forall \phi \in H_{p e r}^{s} .
$$

Así, obtenemos:

Observación 3.3. Se verifica (3.14). En fecto, con la observación 3.1 tendríamos que $\{T(t)\}_{t \in \mathbb{R}}$ es un grupo de clase $C_{0}$. Así, por la observación (3.2) se tendría (3.14).

Por lo tanto, $\{T(t)\}_{t \in \mathbb{R}}$ es un grupo unitario de clase $C_{o}$ en $H_{p e r}^{s}$.

Sean $\varphi_{1}$ у $\varphi_{2}$ datos próximos en $H_{\text {per }}^{s}$, entonces probaremos que sus correspondientes $T(\cdot) \varphi_{1}$ y $T(\cdot) \varphi_{2}$, respectivamente, también están próximos. En efecto, como $\{T(t)\}_{t \in \mathbb{R}}$ es unitario, para $t \in \mathbb{R}$ obtenemos

$$
\left\|T(t) \varphi_{1}-T(t) \varphi_{2}\right\|_{H_{p e r}^{s}}=\left\|T(t)\left[\varphi_{1}-\varphi_{2}\right]\right\|_{H_{p e r}^{s}}=\left\|\varphi_{1}-\varphi_{2}\right\|_{H_{p e r}^{s}} .
$$

Tomando supremo sobre $\mathbb{R}$, tenemos

$$
\sup _{t \in \mathbb{R}}\left\|T(t) \varphi_{1}-T(t) \varphi_{2}\right\|_{H_{p e r}^{s}}=\left\|\varphi_{1}-\varphi_{2}\right\|_{H_{p e r}^{s}} .
$$

De aquí tenemos que si $\varphi_{1} \rightarrow \varphi_{2}$ entonces $T(\cdot) \varphi_{1} \rightarrow T(\cdot) \varphi_{2}$.

A seguir enunciaremos el Teorema 3.1 en función del grupo $\{T(t)\}_{t \in \mathbb{R}}$.

Teorema 3.3. Sea $s \in \mathbb{R} y\{T(t)\}_{t \in \mathbb{R}}$ el grupo unitario de clase $C_{0}$ del Teorema 3.2, $T(\cdot) \phi$ es la única solución de

$$
\mid \begin{aligned}
& u \in C\left(\mathbb{R}, H_{\text {per }}^{s}\right) \cap C^{1}\left(\mathbb{R}, H_{\text {per }}^{s-2}\right) \\
& u_{t}=A_{1} \text { uen } H_{\text {per }}^{s-2} \\
& u(0)=\phi \in H_{\text {per }}^{s}
\end{aligned},
$$

en el sentido que

$$
\lim _{h \rightarrow 0}\left\|\frac{T(t+h) \phi-T(t) \phi}{h}-A_{1} T(t) \phi\right\|_{H_{\text {per }}^{s-2}}=0,
$$

donde $A_{1}:=i \partial_{x}^{2}$, y si $\varphi_{1} \sim \varphi_{2}$ entonces $T(\cdot) \varphi_{1} \sim T(\cdot) \varphi_{2}$.

Además, se satisface la siguiente regularidad: Si $\phi \in H_{\text {per }}^{s}$ entonces $T(t) \phi \in H_{\text {per }}^{r} \forall t \in \mathbb{R}, \forall r \leq s$ y así existe una constante $C>0$ tal que $\|T(t) \phi\|_{H_{\text {per }}^{r}} \leq C\|\phi\|_{H_{\text {per }}^{s}} \forall t \in \mathbb{R}, \forall r<s$ y $\|T(t) \phi\|_{H_{\text {per }}^{s}}=\|\phi\|_{H_{\text {per }}^{s}} \forall t \in \mathbb{R}$.

También, se obtiene que la aplicación: $\phi \rightarrow A_{1} T(\cdot) \phi$ que a cada dato $\phi \in H_{\text {per }}^{s}$ le asigna $A_{1} T(\cdot) \phi$, es continua. Esto es,

$$
\left\|A_{1} T(t) \phi-A_{1} T(t) \widetilde{\phi}\right\|_{s-2} \leq\|\phi-\widetilde{\phi}\|_{s}, \forall t \in \mathbb{R} .
$$

Así,

$$
\sup _{t \in \mathbb{R}}\left\|A_{1} T(t) \phi-A_{1} T(t) \widetilde{\phi}\right\|_{s-2} \leq\|\phi-\widetilde{\phi}\|_{s}
$$

Además, $A_{1} T(t) \phi \in H_{p e r}^{\theta}, \forall t \in \mathbb{R}, \forall \theta \leq s-2$, con $\left\|A_{1} T(t) \phi\right\|_{\theta} \leq\|\phi\|_{s}, \forall t \in \mathbb{R}, \forall \theta \leq s-2$. Así,

$$
\sup _{t \in \mathbb{R}}\left\|A_{1} T(t) \phi\right\|_{\theta} \leq\|\phi\|_{s}, \forall \theta \leq s-2 .
$$

Demostración: La prueba de (3.18) es análoga al del item 4 de la prueba del Teorema 3.1. Y la prueba del resto del enunciado también se sigue como la prueba del Teorema 3.1 y como consecuencia del Teorema 3.2. 
4. Análisis de la diferenciabilidad versus datos iniciales. Con la finalidad de enriquecer nuestro estudio, buscaremos el espacio infinito dimensional donde ocurre la diferenciabilidad y su conexión con el dato inicial.

Teorema 4.1. Sea $s \in \mathbb{R}$, vale el límite

$$
\lim _{h \rightarrow 0}\left\|\frac{u(t+h)-u(t)}{h}-i \partial_{x}^{2} u(t)\right\|_{H_{\text {per }}^{s-2}}=0, \quad \forall t \in \mathbb{R}
$$

si y solamente si $\phi \in H_{\text {per }}^{s}$.

Demostración: Sea $\phi \in H_{\text {per }}^{s}$, la prueba lo hacemos análoga a la prueba de diferenciabilidad del teorema 3.1. Sea $s \in \mathbb{R}$, si $\phi \in H_{\text {per }}^{s}$ entonces $u(t)=\sum_{k=-\infty}^{+\infty} e^{-i k^{2} t} \hat{\phi}(k) e^{i k(\cdot)}$ y $u$ es solución de $\left(P_{3}\right)$. Recuerde obtenemos

$$
\begin{aligned}
& \left\|\frac{u(t+h)-u(t)}{h}-i \partial_{x}^{2} u(t)\right\|_{H_{p e r}^{s-2}}^{2} \\
& =2 \pi \sum_{-\infty}^{+\infty}\left(1+k^{2}\right)^{s-2}\left|e^{-i k^{2} t}\right|^{2}\left|\frac{e^{-i k^{2} h}-1}{h}+i k^{2}\right|^{2}|\widehat{\phi}(k)|^{2} \\
& =\left.2 \pi \sum_{-\infty}^{+\infty}\left(1+k^{2}\right)^{s-2}|\underbrace{\frac{e^{-i k^{2} h}-1}{h}+i k^{2}}_{M(h):=}| \widehat{\phi}(k)\right|^{2} .
\end{aligned}
$$

Usando L'Hospital tenemos que $M(h) \rightarrow 0$ cuando $h \rightarrow 0$. Ahora, necesitamos la convergencia uniforme de la serie para habilitar el intercambio de límites. Para ello procederemos mayorando el k-ésimo término de la serie. Para $h>0$, tenemos

$$
\frac{e^{-i k^{2} h}-1}{h}=\frac{1}{h} \int_{0}^{h}\left(e^{-i k^{2} s}\right)_{s} d s
$$

luego, obtenemos

$$
\left|\frac{e^{-i k^{2} h}-1}{h}\right| \leq \frac{1}{|h|} \int_{0}^{h}\left|e^{-i k^{2} s}\right| 1-\left.i k^{2}\left|d s=\frac{1}{|h|}\right| k\right|^{2}|h|=|k|^{2} .
$$

Usando la desigualdad (4.2) procedemos a mayorar $|M(h)|^{2}$ como sigue

$$
|M(h)|^{2} \leq\left\{2|k|^{2}\right\}^{2} \leq 4\left\{1+|k|^{2}\right\}^{2} .
$$

Pasamos a mayorar el k-ésimo término de la serie, usando la estimativa (4.3), conseguimos

$$
I_{k, t, s}:=\left(1+k^{2}\right)^{s-2}|M(h)|^{2}|\widehat{\phi}(k)|^{2} \leq\left(1+k^{2}\right)^{s-2} 4\left(1+k^{2}\right)^{2}|\widehat{\phi}(k)|^{2} \leq\left(1+k^{2}\right)^{s} 4|\widehat{\phi}(k)|^{2},
$$

y como $\|\phi\|_{s}^{2}<\infty$ entonces la serie es convergente.

Así, usando el Teorema M-Test de Weierstrass tenemos que la serie converge uniformemente y por lo tanto es posible intercambiar los límites, consiguiendo

$$
\lim _{h \rightarrow 0}\left\|\frac{u(t+h)-u(t)}{h}-i \partial_{x}^{2} u(t)\right\|_{H_{p e r}^{s-2}}^{2}=\lim _{h \rightarrow 0} 2 \pi \sum_{k=-\infty}^{+\infty} I_{k, t, s}=2 \pi \sum_{k=-\infty}^{+\infty} \underbrace{\lim _{h \rightarrow 0} I_{k, t, s}}_{=0}=0 .
$$

Recíprocamente, si $\operatorname{lím}_{h \rightarrow 0}\left\|\frac{u(t+h)-u(t)}{h}-i \partial_{x}^{2} u(t)\right\|_{H_{p e r}^{s-2}}=0, \forall t \in \mathbb{R}$, probaremos que $\phi \in H_{p e r}^{s}$. En efecto, como en $H_{\text {per }}^{s-2}$ vale $\partial_{t} u(t)=i \partial_{x}^{2} u(t), \forall t \in \mathbb{R}$. En particular, para $t=0$ es válido :

$$
H_{p e r}^{s-2} \ni \partial_{t} u(0)=i \partial_{x}^{2} u(0)=i \partial_{x}^{2} \phi
$$

Luego, $\phi \in H_{\text {per }}^{s}$.

Así, para $t=0$, se cumple el siguiente resultado.

Corolario 4.1. Sea $s \in \mathbb{R}$, vale el límite

$$
\lim _{h \rightarrow 0}\left\|\frac{u(h)-\phi}{h}-i \partial_{x}^{2} \phi\right\|_{H_{p e r}^{s-2}}=0
$$


si y solamente si $\phi \in H_{\text {per }}^{s}$.

En términos del grupo unitario $\{T(t)\}_{t \in \mathbb{R}}$, obtenemos los dos siguientes resultados cuyas pruebas son análogas a las pruebas del teorema 4.1 y corolario 4.1 respectivamente.

Teorema 4.2. Sea $s \in \mathbb{R}$, vale el límite

$$
\lim _{h \rightarrow 0}\left\|\frac{T(t+h) \phi-T(t) \phi}{h}-A_{1} T(t) \phi\right\|_{H_{\text {per }}^{s-2}}=0, \quad \forall t \in \mathbb{R}
$$

si y solamente si $\phi \in H_{\text {per }}^{s}$.

Así, se cumple el siguiente resultado,

Corolario 4.2. Sea $s \in \mathbb{R}$, vale el límite

$$
\lim _{h \rightarrow 0}\left\|\frac{T(h) \phi-\phi}{h}-A_{1} \phi\right\|_{H_{p e r}^{s-2}}=0
$$

si y solamente si $\phi \in H_{\text {per }}^{s}$.

5. Ecuación de Schrödinger no homogénea. Estudiaremos la existencia de solución de la ecuación de Schrödinger no homogénea en espacios de Sobolev periódico.

Teorema 5.1. Sea $s \in \mathbb{R}$ fijado, $F \in C\left([0, T], H_{p e r}^{s}\right), T>0,\{T(t)\}_{t \in \mathbb{R}}$ el grupo unitario de clase $C_{o}$ en $H_{\text {per }}^{s}$ definido en el Teorema 3.2 (en particular es un semigrupo de contracción) y $u_{p}(t):=\int_{0}^{t} T(t-$ $\tau) F(\tau) d \tau$. Entonces

$$
u_{p} \in C\left([0, T], H_{p e r}^{s}\right) \cap C^{1}\left([0, T], H_{p e r}^{s-2}\right)
$$

Además,

$$
u_{p} \in C\left([0, T], H_{p e r}^{r}\right) \cap C^{1}\left([0, T], H_{p e r}^{r-2}\right), \forall r \leq s .
$$

También, en $H_{\text {per }}^{s-2}$ vale:

$$
\partial_{t} u_{p}(t)=F(t)+\underbrace{\int_{0}^{t} \partial_{t} T(t-\tau) F(\tau) d \tau}_{=i \partial_{x}^{2} u_{p}(t)}
$$

esto es, con respecto a la norma de $H_{p e r}^{s-2}$.

Mejor aún, $\partial_{t} u_{p}(t)=F(t)+i \partial_{x}^{2} u_{p}(t)$ con respecto a la norma de $H_{p e r}^{r-2}, \forall r \leq s$. Así, $u_{p}(t)$ satisface:

$$
\begin{array}{l|l}
\left(P_{3, p}\right) & \begin{array}{l}
u_{p} \in C\left([0, T], H_{p e r}^{s}\right) \cap C^{1}\left([0, T], H_{\text {per }}^{s-2}\right) \\
\partial_{t} u_{p}(t)-i \partial_{x}^{2} u_{p}(t)=F(t) \in H_{p e r}^{s-2} \\
u_{p}(0)=0,
\end{array}
\end{array}
$$

con la primera derivada calculada en la norma de $H_{p e r}^{s-2}$.

Demostración: Probaremos que $u_{p}$ es continua. En efecto, para $t<t^{\prime}$ y $\tau \in\left(t, t^{\prime}\right)$, tenemos

$$
\begin{aligned}
& \left\|u_{p}(t)-u_{p}\left(t^{\prime}\right)\right\|_{s} \\
& \quad=\left\|\int_{0}^{t} T(t-\tau) F(\tau) d \tau-\int_{0}^{t^{\prime}} T\left(t^{\prime}-\tau\right) F(\tau) d \tau\right\|_{s} \\
& \quad \leq\left\|\int_{0}^{t}\left\{T(t-\tau)-T\left(t^{\prime}-\tau\right)\right\} F(\tau) d \tau\right\|_{s}+\left\|\int_{t}^{t^{\prime}} T\left(t^{\prime}-\tau\right) F(\tau) d \tau\right\|_{s} \\
& \quad \leq \int_{0}^{t}\left\|\left\{T(t-\tau)-T\left(t^{\prime}-\tau\right)\right\} F(\tau)\right\|_{s} d \tau+\int_{t}^{t^{\prime}}\left\|T\left(t^{\prime}-\tau\right) F(\tau)\right\|_{s} d \tau .
\end{aligned}
$$

Por otro lado, conseguimos

$$
\int_{0}^{t}\left\|\left\{T(t-\tau)-T\left(t^{\prime}-\tau\right)\right\} F(\tau)\right\|_{s} d \tau<\epsilon \int_{0}^{t} d \tau=\epsilon t \leq \epsilon T
$$


siempre que $\left|t-t^{\prime}\right|<\delta$.

También, obtenemos

$$
\begin{aligned}
\int_{t}^{t^{\prime}}\left\|T\left(t^{\prime}-\tau\right) F(\tau)\right\|_{s} d \tau & =\int_{t}^{t^{\prime}}\|F(\tau)\|_{s} d \tau \\
& \leq \sup _{\tau \in\left[t, t^{\prime}\right]}\|F(\tau)\|_{s} \int_{t}^{t^{\prime}} d \tau \\
& \leq\left(t^{\prime}-t\right) \sup _{\tau \in[0, T]}\|F(\tau)\|_{s}
\end{aligned}
$$

Usando (5.2) y (5.3) en (5.1), obtenemos

$$
\lim _{t \rightarrow t^{\prime}}\left\|u_{p}(t)-u_{p}\left(t^{\prime}\right)\right\|_{s}=0
$$

Análogamente, probamos que

$$
\lim _{t \rightarrow t^{\prime}}\left\|u_{p}(t)-u_{p}\left(t^{\prime}\right)\right\|_{r}=0, \forall r \leq s
$$

En segundo lugar, usando la versión generalizada al cálculo diferencial en espacios de Banach, obtenemos en $H_{p e r}^{s-2}$ :

$$
\begin{aligned}
\partial_{t} u_{p}(t) & =T(t-t) F(t)-T(t-0) F(0) \cdot 0+\int_{0}^{t} \partial_{t} T(t-\tau) F(\tau) d \tau \\
& =F(t)+\int_{0}^{t} \partial_{t} T(t-\tau) F(\tau) d \tau=F(t)+i \partial_{x}^{2} u_{p}(t) .
\end{aligned}
$$

Similarmente, vale $\partial_{t} u_{p}(t)=F(t)+\int_{0}^{t} \partial_{t} T(t-\tau) F(\tau) d \tau=F(t)+i \partial_{x}^{2} u_{p}(t)$ en $H_{p e r}^{r-2}, \forall r \leq s$. Ahora probaremos que $\partial_{t} u_{p}$ es continua. Para $t<t^{\prime}$ y $\tau \in\left(t, t^{\prime}\right)$, conseguimos

$$
\begin{aligned}
\left\|\partial_{t} u_{p}(t)-\partial_{t} u_{p}\left(t^{\prime}\right)\right\|_{s-2} & =\left\|F(t)+i \partial_{x}^{2} u_{p}(t)-\left\{F\left(t^{\prime}\right)+i \partial_{x}^{2} u_{p}\left(t^{\prime}\right)\right\}\right\|_{s-2} \\
& \leq\left\|F(t)-F\left(t^{\prime}\right)\right\|_{s-2}+\left\|i \partial_{x}^{2} u_{p}(t)-i \partial_{x}^{2} u_{p}\left(t^{\prime}\right)\right\|_{s-2} \\
& =\left\|F(t)-F\left(t^{\prime}\right)\right\|_{s-2}+\left\|\partial_{x}^{2} u_{p}(t)-\partial_{x}^{2} u_{p}\left(t^{\prime}\right)\right\|_{s-2} \\
& \leq\left\|F(t)-F\left(t^{\prime}\right)\right\|_{s-2}+\left\|u_{p}(t)-u_{p}\left(t^{\prime}\right)\right\|_{s} .
\end{aligned}
$$

Usando la continuidad de $F$ y $u_{p}$ en (5.4), obtenemos

$$
\lim _{t \rightarrow t^{\prime}}\left\|\partial_{t} u_{p}(t)-\partial_{t} u_{p}\left(t^{\prime}\right)\right\|_{s-2}=0 .
$$

Análogamente, probamos que $\lim _{t \rightarrow t^{\prime}}\left\|\partial_{t} u_{p}(t)-\partial_{t} u_{p}\left(t^{\prime}\right)\right\|_{r-2}=0, \forall r \leq s$.

Finalmente, hemos obtenido en $H_{p e r}^{s-2}$ la igualdad: $\partial_{t} u_{p}(t)=F(t)+i \partial_{x}^{2} u_{p}(t)$ y evidentemente $u_{p}(0)=0$.

Teorema 5.2 (Existencia de solución local). Sea $T>0, s \in \mathbb{R}$ fijado, $F \in C\left([0, T], H_{p e r}^{s}\right) y$

$$
\begin{array}{l|l}
\left(P_{3}^{F}\right) & \begin{array}{l}
\partial_{t} u-i \partial_{x}^{2} u=F(t) \in H_{p e r}^{s-2} \\
u(0)=\phi \in H_{p e r}^{s},
\end{array}
\end{array}
$$

entonces $\exists ! u \in C\left([0, T], H_{\text {per }}^{s}\right) \cap C^{1}\left([0, T], H_{\text {per }}^{s-2}\right)$ solución de $\left(P_{3}^{F}\right)$.

Mejor aún, $u \in C\left([0, T], H_{p e r}^{r}\right) \cap C^{1}\left([0, T], H_{p e r}^{r-2}\right), \forall r \leq s$.

Demostración: La prueba lo haremos del siguiente modo:

1. Primero, obtendremos el candidato a solución. Para conseguir esto, aplicamos la transformada de Fourier a la ecuación no homogénea $\left(P_{3}^{F}\right)$ :

$$
\partial_{t} u(t)-i \partial_{x}^{2} u(t)=F(t)
$$

y obtenemos

$$
\partial_{t} \widehat{u}(k, t)=-i k^{2} \widehat{u}(k, t)+\widehat{F}(k, t),
$$


que para cada $k \in \mathbb{Z}$, es una EDO no homogénea con dato inicial $\widehat{u}(k, 0)=\widehat{\phi}(k)$.

Así, planteamos un sistema no acoplado de ecuaciones de primer orden no homogéneo.

$$
\begin{array}{l|l}
\left.\Omega_{k}\right) & \begin{array}{l}
\widehat{u} \in C\left([0, T], l_{s}^{2}(\mathbb{Z})\right) \\
\partial_{t} \widehat{u}(k, t)=-i k^{2} \widehat{u}(k, t)+\widehat{F}(k, t) \\
\widehat{u}(k, 0)=\widehat{\phi}(k) \operatorname{con} \widehat{\phi} \in l_{s}^{2}(\mathbb{Z}),
\end{array}
\end{array}
$$

$\forall k \in \mathbb{Z}$, que resolveremos a continuación.

Sea $k \neq 0$, multiplicando por el factor integrante $e^{i k^{2} t}$ a la ecuación diferencial de $\left(\Omega_{k}\right)$, obtenemos

$$
\partial_{t}\left\{e^{i k^{2} t} \widehat{u}(k, t)\right\}=e^{i k^{2} t} \widehat{F}(k, t),
$$

integrando de 0 a $t$, obtenemos

$$
\int_{0}^{t} \partial_{\tau}\left\{e^{i k^{2} \tau} \widehat{u}(k, \tau)\right\} d \tau=\int_{0}^{t} e^{i k^{2} \tau} \widehat{F}(k, \tau) d \tau,
$$

luego,

$$
e^{i k^{2} t} \widehat{u}(k, t)-\widehat{u}(k, 0)=\int_{0}^{t} e^{i k^{2} \tau} \widehat{F}(k, \tau) d \tau,
$$

esto es,

$$
\begin{aligned}
\widehat{u}(k, t) & =e^{-i k^{2} t} \widehat{\phi}(k)+e^{-i k^{2} t} \int_{0}^{t} e^{i k^{2} \tau} \widehat{F}(k, \tau) d \tau \\
& =e^{-i k^{2} t} \widehat{\phi}(k)+\int_{0}^{t} e^{-i k^{2}(t-\tau)} \widehat{F}(k, \tau) d \tau .
\end{aligned}
$$

Si $k=0$, la EDO no homogénea es

$$
\mid \begin{aligned}
& \partial_{t} \widehat{u}(0, t)=\widehat{F}(0, t) \\
& \widehat{u}(0,0)=\widehat{\phi}(0)
\end{aligned}
$$

Integrando de 0 a $t$, tenemos:

$$
\int_{0}^{t} \partial_{\tau} \widehat{u}(0, \tau) d \tau=\int_{0}^{t} \widehat{F}(0, \tau) d \tau
$$

Luego,

$$
\widehat{u}(0, t)-\widehat{u}(0,0)=\int_{0}^{t} \widehat{F}(0, \tau) d \tau,
$$

esto es,

$$
\widehat{u}(0, t)=\widehat{\phi}(0)+\int_{0}^{t} \widehat{F}(0, \tau) d \tau .
$$

Finalmente,

$$
\widehat{u}(k, t)=e^{-i k^{2} t} \widehat{\phi}(k)+\int_{0}^{t} e^{-i k^{2}(t-\tau)} \widehat{F}(k, \tau) d \tau, \forall k \in \mathbb{Z} .
$$

El candidato a solución de $\left(P_{3}^{F}\right)$ es

$$
\begin{aligned}
u(t) & =\sum_{k=-\infty}^{+\infty} \widehat{u}(k, t) \phi_{k} \\
& =\sum_{k=-\infty}^{+\infty}\left\{e^{-i k^{2} t} \widehat{\phi}(k)+\int_{0}^{t} e^{-i k^{2}(t-\tau)} \widehat{F}(k, \tau) d \tau\right\} \phi_{k} \\
& =\sum_{k=-\infty}^{+\infty} e^{-i k^{2} t} \widehat{\phi}(k) \phi_{k}+\sum_{k=-\infty}^{+\infty} \int_{0}^{t} e^{-i k^{2}(t-\tau)} \widehat{F}(k, \tau) d \tau \phi_{k} \\
& =\sum_{k=-\infty}^{+\infty} e^{-i k^{2} t} \widehat{\phi}(k) \phi_{k}+\int_{0}^{t} \sum_{k=-\infty}^{+\infty} e^{-i k^{2}(t-\tau)} \widehat{F}(k, \tau) \phi_{k} d \tau \\
& =\underbrace{T(t) \phi}_{u_{h}(t):=}+\underbrace{\int_{0}^{t} T(t-\tau) F(\tau) d \tau}_{u_{p}(t):=},
\end{aligned}
$$


donde $u_{h}$ es la solución de la ecuación homogénea de $\left(P_{3}^{F}\right)$ que ya fue probada y $u_{p}$ es la solución particular de $\left(P_{3}^{F}\right)$ con condición nula, que también fue probada en el teorema previo.

2. Observamos que $u(t) \in H_{p e r}^{s}$. Además, como $u_{h}$ y $u_{p}$ pertenecen a $C\left([0, T], H_{p e r}^{s}\right)$, entonces $u=u_{h}+u_{p} \in C\left([0, T], H_{p e r}^{s}\right)$.

Similarmente, como $u_{h}, u_{p} \in C^{1}\left([0, T], H_{\text {per }}^{s-2}\right)$, entonces $u=u_{h}+u_{p} \in C^{1}\left([0, T], H_{\text {per }}^{s-2}\right)$.

Análogamente se prueba que $u=u_{h}+u_{p} \in C\left([0, T], H_{p e r}^{r}\right) \cap C^{1}\left([0, T], H_{p e r}^{r-2}\right), \forall r \leq s$.

También, verificamos que $u(0)=u_{h}(0)+u_{p}(0)=\phi+0=\phi$. Como existen $\partial_{t} u_{h}(t)$ y $\partial_{t} u_{p}(t)$ en $H_{p e r}^{s-2}$, entonces $u(t):=u_{h}(t)+u_{p}(t)$ satisface en $H_{p e r}^{s-2}$ lo siguiente:

$$
\begin{aligned}
\partial_{t} u(t) & =\partial_{t} u_{h}(t)+\partial_{t} u_{p}(t) \\
& =i \partial_{x}^{2} u_{h}(t)+\left\{i \partial_{x}^{2} u_{p}(t)+F(t)\right\} \\
& =i \partial_{x}^{2}\left\{u_{h}(t)+u_{p}(t)\right\}+F(t) \\
& =i \partial_{x}^{2} u(t)+F(t) .
\end{aligned}
$$

Corolario 5.1. La única solución de $\left(P_{3}^{F}\right)$ es

$$
u(x, t)=\sum_{k=-\infty}^{+\infty} e^{-i k^{2} t} \widehat{\phi}(k) e^{i k x}+\int_{0}^{t} \sum_{k=-\infty}^{+\infty} e^{-i k^{2}(t-\tau)} \widehat{F}(k, \tau) e^{i k x} d \tau .
$$

Teorema 5.3. Sea $T>0$ y u la solución de $\left(P_{3}^{F}\right)$, entonces u satisface:

$$
\begin{aligned}
\|u(t)\|_{r} & \leq\|\phi\|_{s}+T\|F\|_{s, \infty}, \forall r \leq s, \forall t \in[0, T], \\
\sup _{t \in[0, T]}\|u(t)\|_{r} & \leq\|\phi\|_{s}+T\|F\|_{s, \infty}, \forall r \leq s .
\end{aligned}
$$

Además, valen las siguientes desigualdades:

$$
\begin{aligned}
\left\|\partial_{t} u(t)\right\|_{r-2} & \leq\|\phi\|_{s}+(1+T)\|F\|_{s, \infty}, \forall r \leq s, \forall t \in[0, T], \\
\sup _{t \in[0, T]}\left\|\partial_{t} u(t)\right\|_{r-2} & \leq\|\phi\|_{s}+(1+T)\|F\|_{s, \infty}, \forall r \leq s,
\end{aligned}
$$

donde $\|F\|_{s, \infty}:=\sup _{t \in[0, T]}\|F(t)\|_{s}$.

Demostración: Sea $u$ la solución de $\left(P_{3}^{F}\right)$, tenemos

$$
\begin{aligned}
\|u(t)\|_{r} & \leq\|T(t) \phi\|_{r}+\left\|\int_{0}^{t} T(t-\tau) F(\tau) d \tau\right\|_{r} \\
& \leq\|T(t) \phi\|_{s}+\left\|\int_{0}^{t} T(t-\tau) F(\tau) d \tau\right\|_{s} \\
& \leq\|\phi\|_{s}+\int_{0}^{t}\|T(t-\tau) F(\tau)\|_{s} d \tau \\
& =\|\phi\|_{s}+\int_{0}^{t}\|F(\tau)\|_{s} d \tau \\
& \leq\|\phi\|_{s}+\sup _{\tau \in[0, T]}\|F(\tau)\|_{s} \underbrace{\int_{0}^{t} d \tau}_{=t \leq T} \\
& \leq\|\phi\|_{s}+T\|F\|_{s, \infty}, \forall r \leq s .
\end{aligned}
$$

Tomando supremo en la desigualdad (5.5) obtenemos

$$
\sup _{t \in[0, T]}\|u(t)\|_{r} \leq\|\phi\|_{s}+T\|F\|_{s, \infty}, \forall r \leq s .
$$

Por otro lado, como vale en $H_{p e r}^{s-2}$ la igualdad $\partial_{t} u(t)=i \partial_{x}^{2} u(t)+F(t)$, usando la desigualdad triangular de la norma $\|\cdot\|_{r-2}$, la inmersión continua de $H_{p e r}^{s-2} \subset H_{p e r}^{r-2} \forall r \leq s$ y que el operador diferencial $\partial_{x}^{2}$ va de $H_{p e r}^{s}$ en $H_{p e r}^{s-2}$, conseguimos

$$
\begin{aligned}
\left\|\partial_{t} u(t)\right\|_{r-2} & =\left\|i \partial_{x}^{2} u(t)+F(t)\right\|_{r-2} \\
& \leq\left\|\partial_{x}^{2} u(t)\right\|_{r-2}+\|F(t)\|_{r-2} \\
& \leq\left\|\partial_{x}^{2} u(t)\right\|_{s-2}+\|F(t)\|_{s-2} \\
& \leq\|u(t)\|_{s}+\|F(t)\|_{s}, \forall r \leq s .
\end{aligned}
$$


Usando (5.5) en (5.6), obtenemos

$$
\begin{aligned}
\left\|\partial_{t} u(t)\right\|_{r-2} & \leq\|\phi\|_{s}+T\|F\|_{s, \infty}+\|F(t)\|_{s} \\
& \leq\|\phi\|_{s}+(1+T)\|F\|_{s, \infty}, \forall r \leq s .
\end{aligned}
$$

Tomando supremo en la desigualdad (5.7), tenemos

$$
\sup _{t \in[0, T]}\left\|\partial_{t} u(t)\right\|_{r-2} \leq\|\phi\|_{s}+(1+T)\|F\|_{s, \infty}, \forall r \leq s .
$$

A continuación, usando el Teorema 5.3 obtenemos algunas desigualdades que evidencian la dependencia continua de la solución de $\left(P_{3}^{F}\right)$ respecto al dato inicial y a la parte no homogenéa de la ecuación.

Corolario 5.2 (Dependencia continua de la solución de $\left(P_{3}^{F}\right)$ ). Sea $T>0$, s un número real fijado, $\varphi_{j} \in H_{\text {per }}^{s}, F_{j} \in C\left([0, T], H_{p e r}^{s}\right) y$ denotamos por $u_{j}$ a la correspondiente solución de $\left(P_{3}^{F_{j}}\right)$ con dato $\varphi_{j}$, para $j=1,2$. Entonces para $r \leq s$ valen las siguientes desigualdades:

$$
\begin{aligned}
\left\|u_{1}(t)-u_{2}(t)\right\|_{r} & \leq\left\|\varphi_{1}-\varphi_{2}\right\|_{s}+T\left\|F_{1}-F_{2}\right\|_{s, \infty}, \\
\sup _{t \in[0, T]}\left\|u_{1}(t)-u_{2}(t)\right\|_{r} & \leq\left\|\varphi_{1}-\varphi_{2}\right\|_{s}+T\left\|F_{1}-F_{2}\right\|_{s, \infty}, \\
\left\|\partial_{t} u_{1}(t)-\partial_{t} u_{2}(t)\right\|_{r-2} & \leq\left\|\varphi_{1}-\varphi_{2}\right\|_{s}+(1+T)\left\|F_{1}-F_{2}\right\|_{s, \infty}, \\
\sup _{t \in[0, T]}\left\|\partial_{t} u_{1}(t)-\partial_{t} u_{2}(t)\right\|_{r-2} & \leq\left\|\varphi_{1}-\varphi_{2}\right\|_{s}+(1+T)\left\|F_{1}-F_{2}\right\|_{s, \infty} .
\end{aligned}
$$

Corolario 5.3 (Unicidad de solución de $\left(P_{3}^{F}\right)$ ). $\left(P_{3}^{F}\right)$ posee una única solución. Demostración:Es consecuencia del Corolario 5.2, haciendo $F_{1}=F_{2}=F$ y $\varphi_{1}=\varphi_{2}=\phi$.

6. Conclusiones. En nuestro estudio de la ecuación de Schrödinger en espacios de Sobolev periódico tanto en el caso homogéneo $\left(P_{3}\right)$ como en el correspondiente problema no homogéneo $\left(P_{3}^{F}\right)$ hemos obtenido importantes resultados, entre los cuales destacamos:

1. Usando la teoría de Fourier, demostramos la existencia y unicidad de solución del modelo $\left(P_{3}\right)$, así como la dependencia continua de la solución respecto al dato inicial.

2. Probamos la regularidad de la solución de $\left(P_{3}\right)$.

3. Introduciendo una familia de operadores, la cual forma un $C_{0}$ - Grupo, reescribimos la solución del problema $\left(P_{3}\right)$, obteniendo resultados más elegantes.

4. En el análisis de diferenciabilidad de la solución versus el dato inicial obtenemos resultados como el saber en que espacio $H_{p e r}^{r}$ existe la derivada $\partial_{t} u(t)=i \partial_{x}^{2}(t)$ y que esto depende mucho del espacio donde se tome el dato inicial.

5. Usando la teoría de Fourier y la teoría de Grupos y Semigrupo probamos la existencia de solución local y unicidad de solución del modelo no homogéneo $\left(P_{3}^{F}\right)$.

6. Obtenemos la dependencia continua de la solución de $\left(P_{3}^{F}\right)$ respecto al dato inicial y a la parte no homogénea del problema.

7. Además, teóricamente hemos conseguido una familia de operadores que forman un $C_{0}$ - Grupo.

ORCID and License

Yolanda Santiago Ayala https:// orcid.org/0000-0003-2516-0871

Santiago Rojas Romero https:// orcid.org/0000-0002-5354-8059

This work is licensed under the Creative Commons - Attribution 4.0 International (CC BY 4.0)

\section{Referencias}

[1] Burq N, Gérard P, Ptzvetkov N. An instability property of the nonlinear Schrödinger Equation on $S^{d}$. Mathematical Research Letters. 2002; 9:323-335.

[2] Cazenave T. An introduction to nonlinear Schrödinger equations. Third Edition. IM-UFRJ, Rio de Janeiro; 1996.

[3] Iorio R, Iorio V. Fourier Analysis and partial differential equation. Cambridge University; 2001.

[4] Killip R, Tao T, Visan M. The cubic nonlinear Schrödinger equation in two dimensions with radial data. J. Eur. Math. Soc. 2009; 11:1203-1258.

[5] Liu Z, Zheng S. Exponential stability of the semigroup associated with a thermoelastic system. Quarterly of Applied Mathematics. 1993; 51(3):535-545.

[6] Moore W. Schrödinger - Life and Thought. Cambridge University Press; 1989. 
[7] Muñoz Rivera J, Grazia Naso M. Optimal energy decay rate for a class of weakly dissipative second-order systems with memory. Applied Mathematics Letters. 2010; 23:743-746.

[8] Pazy A. Semigroups of linear operator and applications to partial differential equations. Applied Mathematical Sciences. 44 Springer Verlag. Berlín; 1983.

[9] Santiago Y. Sobre la analiticidad del semigrupo $C_{o}$ asociado a un sistema viscoelástico. Pesquimat. 2003; 06(02):27-36.

[10] Santiago Y. Global existence and exponential stability for a coupled wave system. Journal of Mathematical Sciences: Advances and Applications. 2012; 16(1-2):29-46.

[11] Santiago Y. Tópicos de Análisis Funcional. Fundamentos y Aplicaciones. Editorial Académica Española, Alemania; 2014.

[12] Santiago Y, Rojas S. Regularity and wellposedness of a problem to one parameter and its behavior at the limit. Bulletin of the Allahabad Mathematical Society. 2017; 32(2):207-230.

[13] Santiago Y, Rojas S. Existencia y Regularidad de solución de la ecuación del calor en espacios de Sobolev Periódico. Selecciones Matemáticas. 2019; 06(01):49-65.

[14] Schrödinger E. An Undulatory Theory of the Mechanics of Atoms and Molecules. Phys. Rev. 1926; 28:10-49.

[15] Sulem C, Sulem P. The nonlinear Schrödinger equation: self-focusing and wave collapse. Springer Verlag, New York; 1999. 